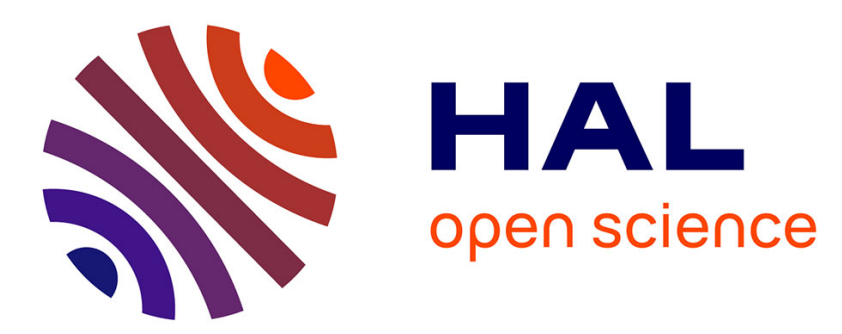

\title{
Influence des effets non linéaires sur la focalisation des faisceaux lasers de haute densité de puissance
}

\author{
J.C. Guyot, A. Bettinger, D. Auric
}

\section{To cite this version:}

J.C. Guyot, A. Bettinger, D. Auric. Influence des effets non linéaires sur la focalisation des faisceaux lasers de haute densité de puissance. Revue de Physique Appliquée, 1978, 13 (4), pp.198-210. 10.1051/rphysap:01978001304019800 . jpa-00244441

\section{HAL Id: jpa-00244441 https://hal.science/jpa-00244441}

Submitted on 1 Jan 1978

HAL is a multi-disciplinary open access archive for the deposit and dissemination of scientific research documents, whether they are published or not. The documents may come from teaching and research institutions in France or abroad, or from public or private research centers.
L'archive ouverte pluridisciplinaire HAL, est destinée au dépôt et à la diffusion de documents scientifiques de niveau recherche, publiés ou non, émanant des établissements d'enseignement et de recherche français ou étrangers, des laboratoires publics ou privés. 
Classification

Physics Abstracts

$42.65-42.70 \mathrm{C}$

\title{
INFLUENCE DES EFFETS NON LINÉAIRES SUR LA FOCALISATION DES FAISCEAUX LASERS DE HAUTE DENSITÉ DE PUISSANCE (*)
}

\author{
J. C. GUYOT, A. BETTINGER et D. AURIC \\ Division Lasers, Laboratoires de Marcoussis, \\ Centre de Recherches de la C. G. E., Route de Nozay, 91460 Marcoussis, France
}

(Reçu le 28 décembre 1976, révisé le 3 janvier 1978, accepté le 11 janvier 1978)

\begin{abstract}
Résumé. - L'intégrale de rupture B exprime le déphasage que subit la surface d'une onde laser traversant un milieu d'indice de réfraction non linéaire élevé $\left(\simeq 10^{-13}\right.$ e. $\mathrm{s}$. u.). Aux densités de puissance importantes (quelques $\mathrm{GW} / \mathrm{cm}^{2}$ ), l'amplitude des modulations d'énergie ayant une fréquence spatiale privilégiée $\left(40\right.$ à $\left.150 \mathrm{~cm}^{-1}\right)$ croît avec un gain égal à $\exp (B)$. Au foyer d'une lentille, l'énergie contenue dans ces modulations ( 20 à $30 \%$ de l'énergie totale lorsque $B>4,5$ ) est rejetée dans des angles supérieurs à 5 fois l'angle limite de diffraction. En outre, la brillance du laser est profondément affectée lorsque $B>7$, et la densité de puissance au foyer peut être minimum lorsque la puissance délivrée par le laser est maximum.
\end{abstract}

\begin{abstract}
A laser beam wave-front going through a medium with a high non-linear index of refraction $\left(\simeq 10^{-13}\right.$ e. s. u. $)$ is affected by a phase shift measured by the beam-break-up integral B. For high power densities (a few $\mathrm{GW} / \mathrm{cm}^{2}$ ), energy ripples with critical spatial frequencies (between 40 and $150 \mathrm{~cm}^{-1}$ ) grow with a gain equal to $\exp (B)$. Energy contained in these ripples is focused outside of an angle equal to 5 times the diffraction angle and represents $20 \%$ to $30 \%$ of the total laser energy when $B>4.5$. Moreover, the laser radiance is deeply disturbed when $B>7$ and the power density at the lens focus can be at its lowest value when the power delivered by the laser reaches a peak value.
\end{abstract}

Introduction. - Les résultats les plus significatifs, issus des expériences d'interaction lumière-matière, ont été obtenus, jusqu'à ce jour, en utilisant le laser à verre dopé au néodyme. Ces expériences sont orientées, en particulier, vers la production d'énergie au moyen de réactions thermonucléaires induites par laser.

Le laser à verre dopé au néodyme présente, en effet, des caractéristiques particulièrement intéressantes qui expliquent le développement qu'il a connu au cours de ces dix dernières années : citons, par exemple, sa longueur d'onde d'émission, proche du visible, sa section efficace d'émission stimulée, qui permet un stockage d'énergie aisé, la possibilité de produire des impulsions courtes, résultant d'une largeur spectrale de fluorescence suffisamment importante et, enfin, la possibilité d'élaboration de pièces de verre de grande dimension.

Mais c'est le verre qui nous impose les limites d'utilisation de ce laser, limites qui sont différentes

(*) Cet exposé traite, en particulier, de travaux effectués avec l'appui du C. E. A., Division des Applications Militaires, présenté au Congrès National de Physique des Plasmas, Paris 6-10 décembre 1976. selon que l'on désire en extraire de l'énergie ou de la puissance. Lorsqu'on désire extraire de l'énergie du laser (1 kJ dans des impulsions voisines de $1 \mathrm{~ns})$, la limitation est imposée par le seuil de tenue de la matrice de verre (de l'ordre de $10 \mathrm{~J} / \mathrm{cm}^{2}$ ). Par contre, lorsqu'on demande au laser de délivrer de la puissance en des temps courts ( $1 \mathrm{TW}$ dans des impulsions de $0,1 \mathrm{~ns}$ ), les densités de puissance dans le matériau sont de l'ordre de plusieurs $\mathrm{GW} / \mathrm{cm}^{2}$ : l'onde laser induit alors, dans le verre, des champs électriques suffisamment élevés pour modifier sa structure et faire varier, en particulier, son indice de réfraction.

Dans ce qui suit, nous nous proposons de montrer :

- comment l'influence du champ électrique de l'onde laser sur l'indice de réfraction du verre conduit à limiter la puissance que l'on peut extraire d'un laser à verre et conditionne les valeurs de densité de puissance qu'il est possible de focaliser sur une cible de petite dimension;

- comment ce phénomène intervient dans le choix des composants laser et leur disposition dans une chaîne de puissance. 
1. Autofocalisation en milieu non linéaire. - L'indice de réfraction du verre laser, en présence d'un champ électrique $E(r, z, t)$ s'écrit:

$$
n(r, z, t)=n_{0}+n_{2} \cdot E^{2}(r, z, t)
$$

où $n_{0}$ est l'indice habituel du verre, et $n_{2}$ est l'indice non linéaire.

Dans un morceau de verre homogène, $n_{0}$ et $n_{2}$ sont indépendants du temps et de l'espace. Par contre, lorsque ce morceau de verre est traversé par un faisceau laser intense, l'indice $n$ vu par l'onde laser est fonction de sa puissance, et la propagation du faisceau laser dépendra donc de sa propre structure spatiale et temporelle : il y a autofocalisation du faisceau laser.

La variation d'indice induite par l'onde laser s'écrit :

$$
\Delta n(\mathbf{r}, z, t)=n-n_{0}=\gamma \cdot I(\mathbf{r}, z, t)
$$

où $I$ est la densité de puissance de l'onde $\left(\mathrm{W} / \mathrm{cm}^{2}\right)$

et

$$
\gamma=4,19 \times 10^{-3} \frac{n_{2}}{n_{0}},
$$

$n_{2}$ étant exprimé en e. s. u. et ayant, dans le cas du verre laser, $n_{2}=10^{-13}$ e. s. u., comme ordre de grandeur typique.

Il en résulte [1] que, en un point $Z$ de l'axe de propagation du laser, la surface d'onde sera affectée d'un retard de phase $\Delta \varphi(\mathbf{r}, t)$

$$
\Delta \varphi(\mathbf{r}, t)=k \int_{0}^{Z} \Delta n \mathrm{~d} z=k \gamma \int_{0}^{Z} I(\mathbf{r}, z, t) \mathrm{d} z
$$

$k=2 \pi / \lambda$ et représente le nombre d'onde dans le vide.

On appelle intégrale de rupture (beam break-up integral) la quantité :

$$
B(\mathbf{r}, t)=k \gamma \int_{0}^{Z} I(\mathbf{r}, z, t) \mathrm{d} z .
$$

La relation (3) montre que la qualité de l'onde, donc ses propriétés au foyer d'une lentille, seront variables durant le temps de l'impulsion et dépendront aussi de la répartition spatiale $I(\mathbf{r})$ de la puissance.

Généralement, on peut considérer $I(\mathbf{r})$ comme la somme :

- d'une enveloppe moyenne $I_{0}(\mathbf{r})$, ayant des composantes spectrales en $r$ suffisamment basses pour ne pas être déformée par diffraction dans la chaîne laser,

- d'une modulation $\Delta I(\mathbf{r})$ contenant des fréquences spatiales élevées sur lesquelles interviennent simultanément la diffraction et la variation non linéaire de l'indice

$$
I(\mathbf{r})=I_{0}(\mathbf{r})+\Delta I(\mathbf{r}) .
$$

Lorsque $\Delta I(\mathbf{r})=0$, nous sommes en présence du phénomène d'autofocalisation globale du faisceau laser [2], qui a pour effet d'introduire, en champ proche, une distorsion de phase sur l'onde laser, ce qui se traduira, en champ lointain, par une modification de la répartition d'énergie dans la tache focale (phénomène d'aberration).

Par contre, les modulations $\Delta I(\mathbf{r})$ à petite échelle seront amplifiées au cours de leur propagation dans le verre laser $[3,4,5]$; il en résultera une onde fortement perturbée en sortie du laser et, en champ lointain, la puissance contenue dans ces perturbations sera dispersée tout autour de la tache focale : elle ne contribuera pas à l'augmentation de la densité de puissance focalisée $[6,7]$.

2. Autofocalisation à petite échelle. - 2.1 RésulTATS FONDAMENTAUX EN MILIEU PASSIF. - L'analyse mathématique repose sur une étude de la relaxation d'une perturbation spatiale imposée à une onde plane se propageant dans un milieu non linéaire (voir Annexe A). De cette analyse, il se dégage les résultats suivants :

a) Pour certaines valeurs de la fréquence spatiale de la perturbation (voir équation (A.20)), celle-ci croît exponentiellement avec la distance de propagation.

b) Il existe une fréquence spatiale critique $K_{\max }$ de cette perturbation (équation (A.23)), dont la valeur est proportionnelle à la racine carrée de la densité de puissance. A cette fréquence critique, la perturbation aura un gain maximum, qui, après traversée d'une longueur $L$ dans le milieu non-linéaire, aura atteint une valeur $G\left(K_{\max }\right)$ telle que :

$$
G\left(K_{\max }\right)=\exp B\left(K_{\max }\right)
$$

avec

$$
B\left(K_{\max }\right)=k \gamma I_{0} \cdot L
$$

où $I_{0}$ est la densité de puissance du faisceau laser,

$L$ la longueur de verre traversée,

$k \gamma$ le coefficient caractéristique du verre dans lequel interviennent la longueur d'onde laser, l'indice $n_{0}$ et l'indice non linéaire $n_{2}$ du verre (équation (A.24)).

Le coefficient de gain $B$ de la perturbation critique $K_{\max }$ est proportionnel au produit intensité $\times$ longueur de verre traversée.

Une vérification de ces résultats théoriques a été entreprise à l'aide du montage expérimental décrit sur la figure 1. Un oscillateur YAG fournit une impulsion de $80 \mathrm{ps}$ : après passage dans un filtrage spatial, destiné à éliminer les modulations d'intensité, le faisceau est amplifié à bas niveau, jusqu'à un système afocal où l'intensité peut alors croître jusqu'à $10 \mathrm{GW} / \mathrm{cm}^{2}$. Grâce à un système de coin d'air, on peut superposer au faisceau un système de franges, simulant une perturbation mono-dimensionnelle. On compare les modulations du faisceau avant et après passage dans le milieu test (qui est un barreau de verre laser passif), en fonction de l'espace inter-franges, qui représente la fréquence spatiale de la perturbation. 


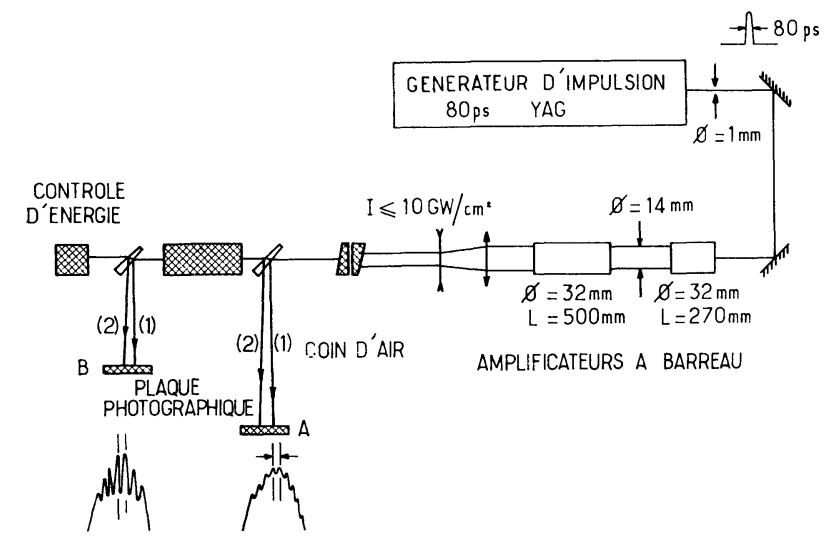

Fig. 1. - Dispositif expérimental de mesure du gain des perturbations.

[Experimental set-up for measuring the gain of perturbations.]

Les résultats obtenus $[8,9]$, dans le cas où le milieu test est un barreau, sont représentés sur la figure 2 et l'on dénote un assez bon accord avec les prédictions théoriques. Aux densités de puissance couramment utilisées (quelques $\mathrm{GW} / \mathrm{cm}^{2}$ ), les fréquences critiques sont comprises entre 100 et $150 \mathrm{~cm}^{-1}$ (correspondant à des dimensions de 0,4 à $0,6 \mathrm{~mm}$ ).

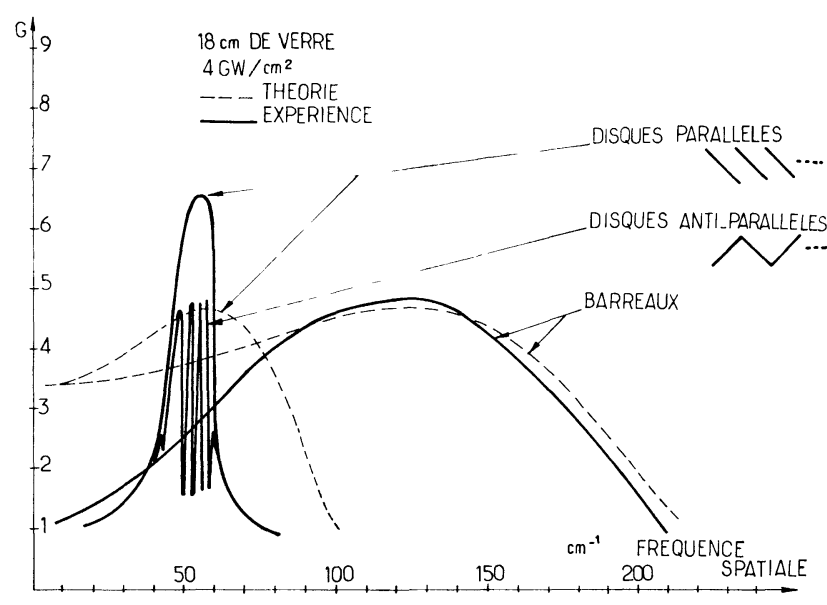

Fig. 2. - Gain des perturbations en fonction de leur fréquence spatiale $(q=2 \pi / \Lambda)$. Comparaison entre une structure à barreau et une structure à disques.

[Gain of perturbations vs. their spatial frequency $(q=2 \pi / \lambda)$. Comparison between rod and disk structures.]

Ces expériences ont été étendues $[10,11]$ au cas d'un milieu test discontinu (amplificateur à disques) et ont, alors, fait apparaître des différences significatives :

- les fréquences critiques se situent vers des valeurs plus basses, aux alentours de $40 \mathrm{~cm}^{-1}$ (correspondant à une dimension de $1,5 \mathrm{~mm}$ ) ;

- à ces fréquences, et à densité de puissance égale, le gain des perturbations est plus élevé ;

- la bande passante est plus étroite.
Les prévisions théoriques faites, dans le cas du milieu discontinu, utilisent un formalisme mathématique mieux adapté à cette géométrie [5]; elles conduisent à des valeurs de fréquence critique qui sont correctes par rapport aux valeurs expérimentales; les valeurs de gain semblent, cependant, sous-estimées.

Ce même formalisme peut, évidemment, être utilisé pour déterminer le spectre de fréquence d'une chaîne laser complète.

2.2 Autofocalisation a petite échelle Dans UN AMPLIFICATEUR DE PUISSANCE. - 2.2.1 Intégrale de rupture. - Les résultats théoriques et expérimentaux formulés jusqu'à présent sont valables lorsque le milieu est passif. Si le milieu est actif (présence d'une inversion de population, donc amplification de l'onde laser), le gain de la perturbation $G\left(K_{\max }\right)$ à la fréquence critique peut être obtenu, en première approximation, en remplaçant la densité de puissance $I_{0}$ (7) par la densité de puissance moyenne $I_{\mathrm{m}}$ le long de l'axe de propagation $Z$ de l'amplificateur, de longueur $L$ :

$$
I_{\mathrm{m}}=\frac{1}{L} \int_{0}^{L} I(z) \mathrm{d} z
$$

Le coefficient de gain de la perturbation $B\left(K_{\max }\right)$, à la fréquence critique, sera alors obtenu par la relation (7), en remplaçant le produit $I_{0} . L$ par $I_{\mathrm{m}} L$

$$
B\left(K_{\max }\right)=k \gamma \int_{0}^{L} I(z) \mathrm{d} z .
$$

On retrouve une forme particulière de l'intégrale de rupture définie par la relation (4).

2.2.2 Densité de puissance limite en sortie d'un système amplificateur. - La densité de puissance que l'on peut obtenir en sortie d'un système amplificateur va dépendre de l'amplification que vont subir les perturbations à la traversée d'un tel système. Autrement dit, la performance du système va être limitée par la croissance des instabilités existant sur le faisceau.

Considérons, en effet, un système amplificateur ayant un coefficient de gain $a$, une longueur $L$ et donc un gain :

$$
g=\exp (a L)
$$

où $a\left(\mathrm{en}^{-1}\right)$ est tel que

$$
a=\sigma . \Delta N
$$

$\sigma:$ section efficace d'émission stimulée,

$\Delta N$ : inversion de population.

Si l'on néglige, en première approximation, les effets de saturation du gain (cette approximation est valable pour des impulsions courtes (ps) où les densités d'énergie sont plus éloignées de la valeur de densité d'énergie de saturation que pour des impulsions longues (ns)), la densité de puissance est telle que :

$$
I(L)=I_{\mathrm{e}} g
$$


où $I_{\mathrm{e}}$ est la densité de puissance à l'entrée du système amplificateur. L'intégrale de rupture s'exprime alors :

$$
B\left(K_{\max }\right)=k \gamma \frac{I_{\mathrm{s}}-I_{\mathrm{e}}}{a}=k \gamma \frac{I_{\mathrm{s}}}{a}\left(1-\frac{1}{g}\right)
$$

où $I_{\mathrm{s}}$ est la densité de puissance en sortie du système amplificateur.

Si le gain du système est important $(g \gg 1)$, la densité de puissance maximum que l'on pourra obtenir en sortie du système est donnée par :

$$
\left(I_{\mathrm{s}}\right)_{\mathrm{lim}}=\frac{1}{k \gamma} a\left(B\left(K_{\max }\right)\right)_{\mathrm{lim}}
$$

où $\left(B\left(K_{\max }\right)\right)_{\text {lim }}$ est la valeur maximum de l'intégrale de rupture que l'on peut tolérer pour le système amplificateur.

La formule (14) montre que $\left(I_{\mathrm{s}}\right)_{1 \mathrm{im}}$ est proportionnelle à

$$
\left(\lambda_{0} n_{0} \sigma / n_{2}\right) \Delta N . B_{1 \mathrm{im}} .
$$

La quantité $n_{0} \sigma / n_{2}$ caractérise le verre laser utilisé, $\Delta N$ l'inversion de population dans l'amplificateur. Dans le paragraphe suivant, nous montrons que $(B)_{\lim }$ dépend de la qualité du faisceau laser.

\subsubsection{Valeur maximum de l'intégrale de rupture. -} Il est donc d'une importance primordiale de connaître la valeur maximale de l'intégrale de rupture que l'on peut tolérer dans un amplificateur. Cette valeur peut être estimée de façon théorique : en effet, la théorie des perturbations développée jusqu'à présent s'est limitée à un calcul du premier ordre, ce qui suppose que la croissance de la perturbation est restée suffisamment faible pour qu'elle n'interagisse pas avec elle-même.

Au-delà de ce cas, on peut effectuer un calcul au second ordre (voir $\S \mathrm{A}$.II de l'annexe A) qui indique que l'on doit respecter la condition

$$
\delta \cdot \exp \left[B\left(K_{\max }\right)\right] \leqslant 1
$$

pour rester dans un domaine où la croissance de la perturbation est limitée.

La constante $\delta$ représente la valeur initiale de l'amplitude de la perturbation et la condition (15) exprime, physiquement, que le produit du bruit par son amplification doit rester inférieur à une certaine limite.

La valeur maximum de l'intégrale de rupture sera donc atteinte lorsque :

$$
\delta \cdot \exp \left[\left(B\left(K_{\max }\right)\right)_{1 \mathrm{im}}\right]=1 .
$$

Cette dernière équation fixe donc la valeur maximum du gain de perturbation que l'on peut tolérer dans un système amplificateur si l'on veut éviter l'autofocalisation.

2.3 ANALYSE EN CHAMP PROCHE. - Une analyse en champ proche du faisceau laser permet de dégager une première valeur maximum de l'intégrale de rup- ture. En effet (voir annexe B), on peut définir un indice de modulation $m$ tel que :

$$
m=\frac{I_{0}+\Delta I}{I_{0}}=\left(1+\delta \cdot \exp \left[B\left(K_{\max }\right)\right]\right)^{2}
$$

où $I_{0}$ représente la densité de puissance moyenne du faisceau laser et $\Delta I$ les modulations de cette densité, mesurées à partir de $I_{0}$.

On conclut que, lorsque la valeur limite donnée par l'équation (16) sera atteinte, on aura :

$$
m=4 \text { (ou } 6 \mathrm{~dB}) \text {. }
$$

L'indice de modulation est une valeur susceptible d'être mesurée expérimentalement (densitométrie de plaques photographiques impressionnées par le faisceau laser). La figure 3 indique le schéma d'une chaîne

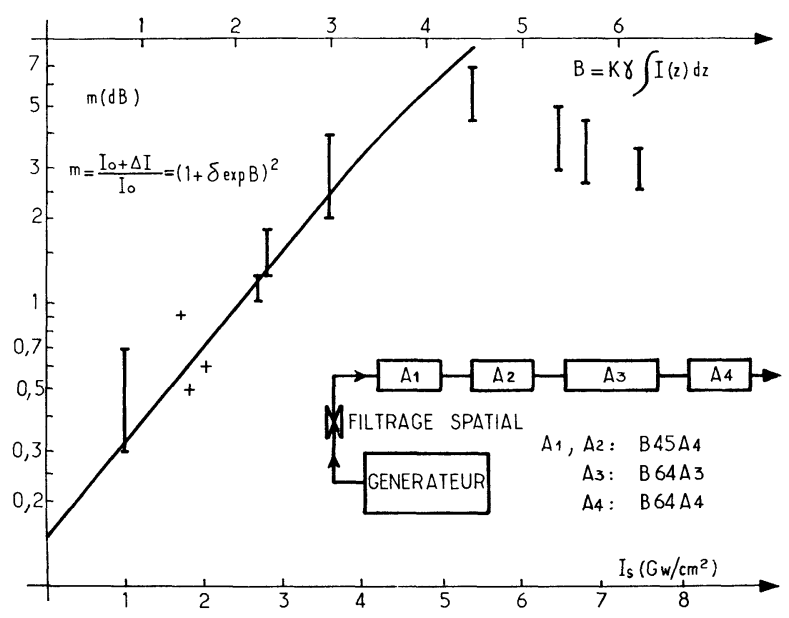

Fig. 3. - Analyse du faisceau en champ proche. Croissance des modulations en fonction de l'intégrale de rupture $B$.

[Near-field beam analysis. Modulation growth-rate vs. B.]

de grande puissance utilisée pour mesurer l'indice de modulation d'un faisceau qui a subi une amplification, après avoir traversé un filtrage spatial situé en entrée de chaîne. Le rôle du filtrage spatial est d'éliminer, en sortie de générateur, les imperfections les plus marquantes du faisceau et d'assurer une répartition spatiale de l'énergie aussi lisse que possible.

Sur cette même figure 3 , on remarque que la variation de l'indice de modulation $m$ en fonction de l'intégrale de rupture $B\left(K_{\max }\right)$ coïncide de façon satisfaisante avec les points expérimentaux jusqu'à une valeur de $B\left(K_{\max }\right)$ comprise entre 4 et 5 . Au-delà, apparaît un désaccord qui traduit le fait que la théorie linéaire ne rend plus compte des phénomènes : nous avons alors atteint la région où la croissance des perturbations conduira à la focalisation des pointes de modulation et donc à des fractures en filaments à l'intérieur du verre laser.

La valeur maximum de $B\left(K_{\max }\right)$ que l'on peut tolérer est donc d'environ 4,5. Dans ces conditions, on déduit de l'équation (16) que $\delta \simeq 1,1 \times 10^{-2}$. 
On constate que la valeur obtenue en prolongeant la courbe jusqu'à $B\left(K_{\max }\right)=0$ est $\delta=1,6 \times 10^{-2}$.

En conclusion, il existe une assez bonne confirmation expérimentale, déduite de mesures effectuées en champ proche, de la validité de la relation (15) comme critère à respecter pour demeurer en-deçà de l'autofocalisation catastrophique. On déduit, en outre, que pour une chaîne pré-amplificatrice (c'est-à-dire un tronçon amplificateur qui fait suite à un filtrage spatial à bas flux) à l'entrée de laquelle se trouve un faisceau dont la qualité est particulièrement bonne :

$$
\begin{gathered}
\delta \simeq 10^{-2} \\
{\left[B\left(K_{\max }\right)\right]_{\mathrm{lim}} \simeq 4,5 .}
\end{gathered}
$$

Il en résulte aussi que, si l'on veut poursuivre l'amplification d'un faisceau ayant atteint la valeur $B_{\max } \simeq 4,5$, il est indispensable de réduire, ou mieux de supprimer, les modulations existantes. Cette opération est effectuée dans les chaînes de puissance au moyen d'un filtrage spatial à haut flux. L'effet d'un tel filtre est montré sur la figure 4 : le faisceau est focalisé à travers un diaphragme, situé dans le plan focal de la lentille d'entrée ; ce diaphragme élimine les modulations à haute fréquence. En sortie du filtrage, le faisceau laser est débarrassé des pointes de modulations : seules subsistent des perturbations résiduelles à basse fré- quence correspondant à la fréquence de coupure du diaphragme. Les caractéristiques de fonctionnement d'un tel filtrage découlent de l'étude en champ lointain du faisceau laser perturbé.

2.4 Analyse en Champ LoIntain. - 2.4.1 Rendement de focalisation. - En sortie d'un système amplificateur, l'expression du champ électrique résultant du calcul de perturbation limité au premier ordre est traduite par les équations (B.1) et (B.2) (voir annexe B).

En effectuant une transformation de Fourier de ce champ électrique, il est possible [6] de calculer la densité de puissance obtenue au foyer d'une lentille parfaite.

On constate que la figure de diffraction ainsi obtenue se compose d'une tache centrale telle qu'elle serait obtenue à partir d'une pupille uniformément éclairée par une onde plane (tache d'Airy): la densité de puissance contenue dans cette tache a, cependant, été réduite d'un facteur $f(\beta)$ où

$$
\beta=\delta \exp \left[B\left(K_{\max }\right)\right] .
$$

La puissance perdue se répartit dans des lobes annexes et résulte de la diffraction, dans des angles relativement élevés par rapport à la direction de propagation, de l'énergie contenue dans les perturbations.

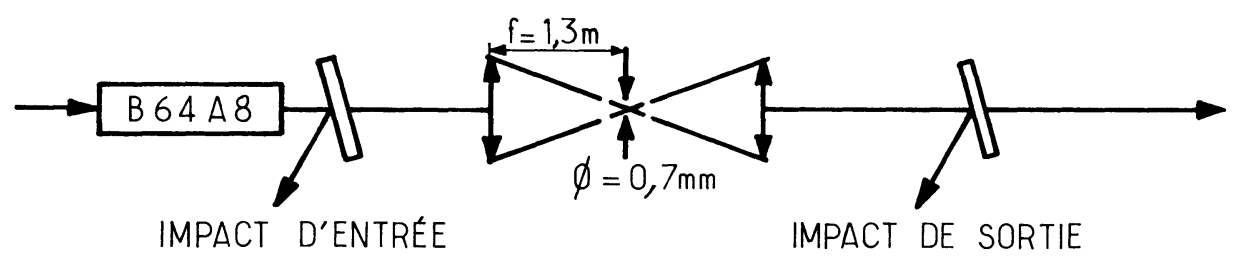

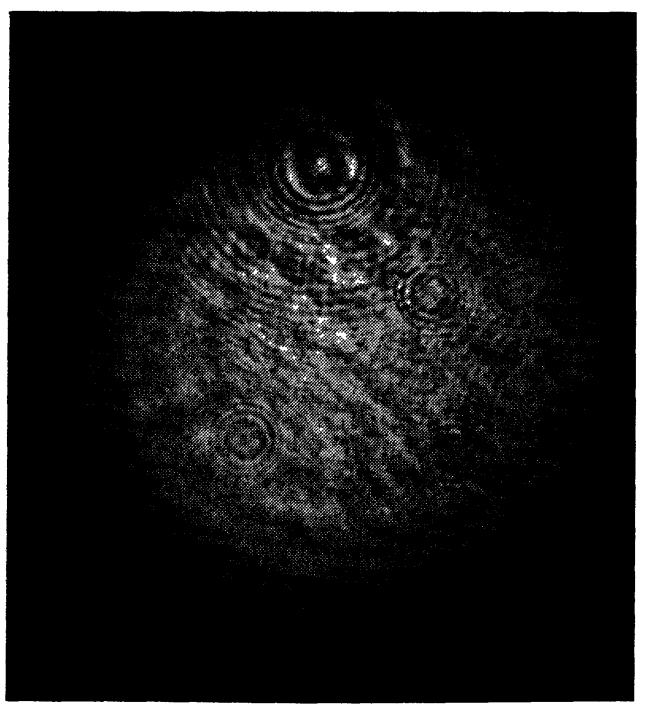

P 50

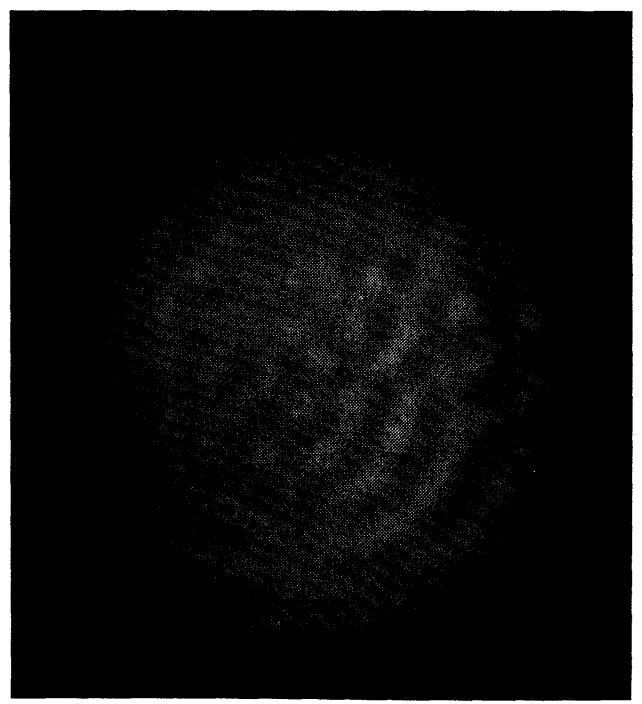

P 50 bis

Fig. 4. - Dispositif de filtrage d'un faisceau laser à haut flux. Comparaison du faisceau en entrée et en sortie de filtrage.

[High-flux spatial filter schematics. Comparison between input and output beam.] 
La figure 5 représente le rendement de focalisation, c'est-à-dire :

$$
P_{\mathrm{F}} / P_{\mathrm{L}}=f(\beta)
$$

où $P_{\mathrm{F}}$ et $P_{\mathrm{L}}$ sont respectivement la puissance focalisée contenue dans la tache d'Airy centrale et la puissance fournie par le laser.

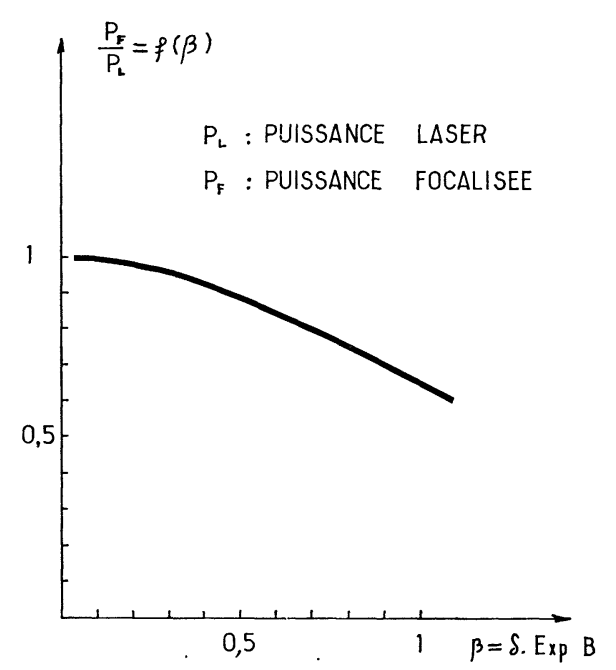

Fig. 5. - Rendement de focalisation. Résultat théorique.

[Focusing efficiency. Theoretical results.]

On note que le rendement de focalisation décroît de façon monotone et que, pour la valeur limite $\delta . \exp B\left(K_{\max }\right)=1$, il atteint environ $70 \%$.

2.4.2 Puissance focalisée. - 2.4.2 a) Théorie. D'après l'équation (22), la puissance focalisée s'écrit :

$$
P_{\mathrm{F}}=f(\beta) P_{\mathrm{L}} \text {. }
$$

Or, l'expression de la puissance fournie par le laser résulte de l'équation (13), où pour $g \gg 1$

$$
P_{\mathrm{L}}=S I_{\mathrm{s}}=\frac{S a}{k \gamma} \cdot B\left(K_{\max }\right)
$$

d'où la relation :

$$
P_{\mathrm{F}}=h B\left(K_{\max }\right) f(\beta) .
$$

La constante $h$ ne dépend que de la structure du laser.

La figure 6 représente la variation de $P_{\mathrm{F}}$ en fonction de $B\left(K_{\max }\right)$ pour deux valeurs de $\delta$.

On notera que, pour chacun des cas, il existe un point optimum de fonctionnement du laser (qui correspond à une puissance focalisée maximum) définie par une valeur de l'intégrale de rupture. Notons que cette valeur optimum de $B$ est très voisine de la valeur $(B)_{\text {lim }}$ déterminée par l'analyse en champ proche (pour $\delta=0,01,(B)_{1 \mathrm{im}} \simeq B_{\mathrm{opt}} \simeq 4,5$ ).

2.4 .2 b) Comparaison avec les résultats expérimentaux. - La figure $7 a$ décrit le montage expérimental utilisé pour mesurer l'énergie $E_{\mathrm{f}}$ focalisée dans

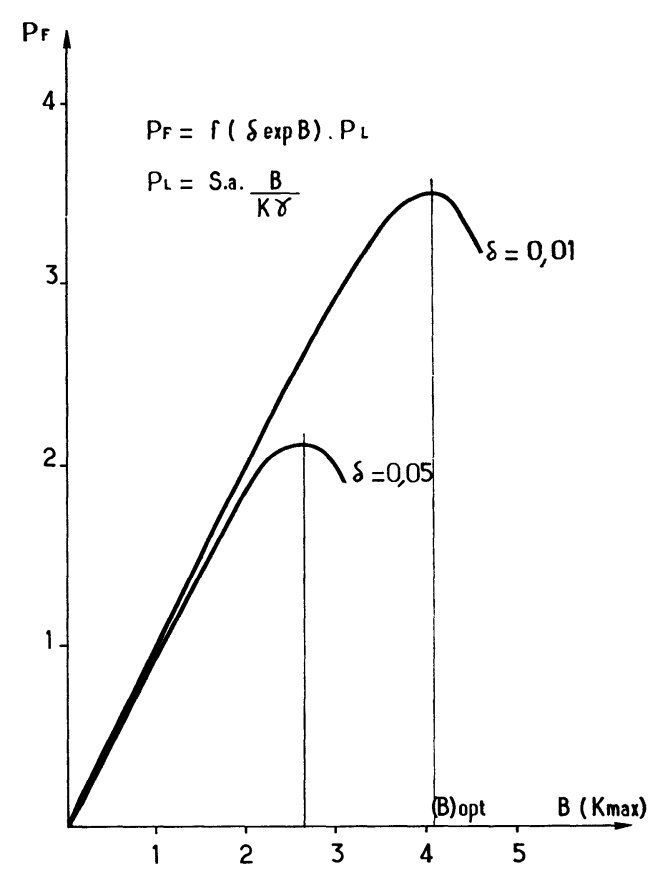

Fig. 6. - Puissance focalisée. Résultat théorique.

[Focused power. Theoretical results.]
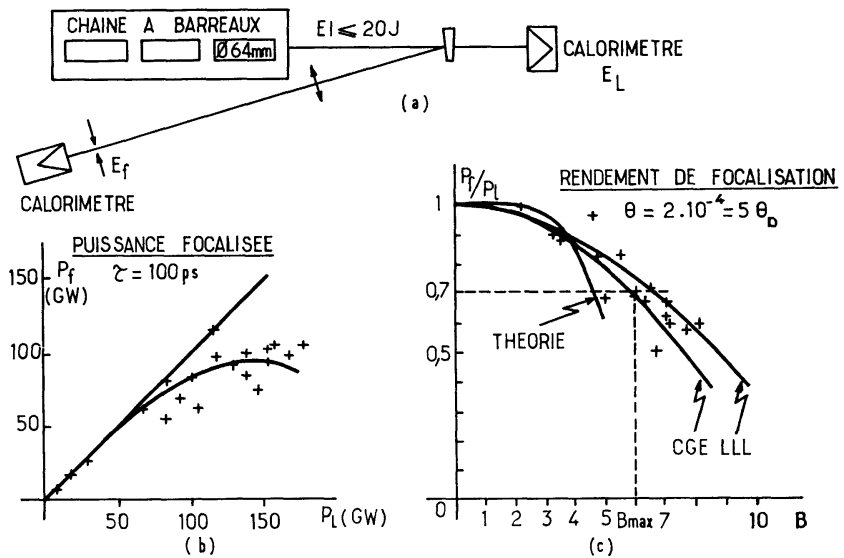

FIG. 7. - a) Montage expérimental destiné à mesurer la puissance focalisée dans un angle égal à environ 5 fois la limite de diffraction. $b$ ) Puissance focalisée en fonction de la puissance émise par le laser. c) Rendement de focalisation en fonction de l'intervalle de rupture. La courbe théorique est déduite de la figure 5 , pour $\delta=0,01$.

[Focusing efficiency and focused power. Experimental results.]

un angle égal à environ 5 fois la limite de diffraction en fonction de l'énergie $E_{\mathrm{L}}$ émise par le laser. La figure $7 b$ traduit les résultats expérimentaux obtenus, à une durée d'impulsion égale à 100 ps. Jusqu'à une puissance $P_{\mathrm{L}}$ d'environ $50 \mathrm{GW}$, les points expérimentaux se situent sur une droite $P_{\mathrm{L}}=P_{\mathrm{F}}$. Au-delà, la puissance focalisée est nettement inférieure à la puissance émise et, dans le cas particulier du montage expérimental utilisé, il serait inutile d'opérer au-delà de $P_{\mathrm{L}}=150 \mathrm{GW}$. La figure $7 c$ est une autre façon de 
mettre en évidence la baisse progressive du rendement de focalisation en fonction de $B\left(K_{\max }\right)$. Les valeurs de rendement de focalisation obtenues tant à Lawrence Livermore Lab. que sur la chaîne expérimentale des Laboratoires de Marcoussis ont des variations pratiquement identiques en fonction de $B\left(K_{\max }\right)$.

Il apparait, en particulier, que la valeur de 0,7 est obtenue pour une intégrale de rupture voisine de 6 , alors que, théoriquement, cette valeur du rendement de focalisation doit correspondre à $B\left(K_{\max }\right) \simeq 4,5$.

Les résultats expérimentaux obtenus en champ lointain semblent indiquer, en première analyse, que les valeurs maximum des intégrales de rupture obtenues à partir d'analyses en champ proche sont, sans doute, quelque peu pessimistes et garantissent, dans une certaine mesure, une marge de sécurité.

Trois remarques d'une extrême importance s'imposent cependant :

a) L'examen des résultats et des impacts obtenus dans le plan focal de la lentille de concentration montre que l'énergie laser focalisée est répartie dans une tache compacte, à forte densité, dont le diamètre est inférieur à 5 fois la limite de diffraction imposée par la pupille de sortie du laser. La partie significative de l'énergie contenue dans les perturbations est répartie tout autour de cette tache centrale, à l'extérieur d'un cercle de rayon égal à 5 fois la limite de diffraction.

b) L'examen approfondi des taches focalisées montre que la distribution d'énergie à l'intérieur de ces taches est très altérée, en particulier pour les valeurs de $B\left(K_{\max }\right)$ élevées (Fig. 8$)$. Ce phénomène est très certainement relié à la distorsion de phase causée par l'autofocalisation globale du faisceau. Pour supprimer les aberrations qui sont ainsi produites, on pourrait être conduit à faire fonctionner le laser avec une valeur de $B\left(K_{\max }\right)$ réduite (2 à 3$)$ ou à compenser les distorsions de phase induites par l'autofocalisation globale.

c) Les résultats expérimentaux obtenus proviennent de mesures calorimétriques. Il faut, en tout état de cause, compléter ces résultats en analysant, en fonction du temps, l'évolution de l'énergie focalisée qui montrera certainement une modification du profil temporel de l'impulsion.

Il résulte de ces remarques que les conditions d'éclairement d'une cible restent à préciser en mesurant l'évolution temporelle de l'énergie focalisée, et ceci pour différentes valeurs de l'intégrale de rupture.

3. Analyse temporelle de la tache focale. Autofocalisation globale. - 3.1 BASES THÉORIQUES. Nous avons vu (c. f. $\S 1$ et Annexe A) que le champ électrique, solution de l'équation de propagation en milieu non linéaire, pouvait être écrit sous la forme :

$$
E=\left(A_{0}+A_{1}\right) \exp i\left(\phi_{0}+\phi_{1}\right)
$$

$A_{0}$ et $\phi_{0}$ représentant l'amplitude et la phase d'une onde plane sur laquelle se superposent des perturbations à petite échelle d'amplitude $A_{1}$ et de phase $\phi_{1}$.

Après propagation dans un milieu non linéaire de longueur $L, A_{1}$ et $\phi_{1}$ sont amplifiés par autofocalisation (relations (A.16) et (A.17)), alors que la composante moyenne de l'amplitude $A_{0}$ reste inchangée. Par contre, la phase $\phi_{0}$ subit un retard $\Delta \varphi$ donné par la relation (3) dont la relation (A.10) n'est qu'un cas particulier (onde plane en régime stationnaire).

Si l'impulsion laser a une distribution temporelle de l'intensité donnée par $Q(t)$, on peut poser

$$
I(\mathbf{r}, z, t)=I(\mathbf{r}, z) Q(t) .
$$

Dans l'hypothèse, généralement vérifiée, où un faisceau de révolution se propage sans modification notable de son profil (ce qui implique que l'inclinaison des rayons reste faible par rapport aux dimensions du faisceau), l'équation (26) peut s'écrire :

$$
I(\mathbf{r}, z, t)=I_{0}(z) F(\mathbf{r}) Q(t)
$$

relation dans laquelle $F(\mathbf{r})$ représente la fonction radiale de l'intensité et $I_{0}(z)$ l'intensité au centre du faisceau.
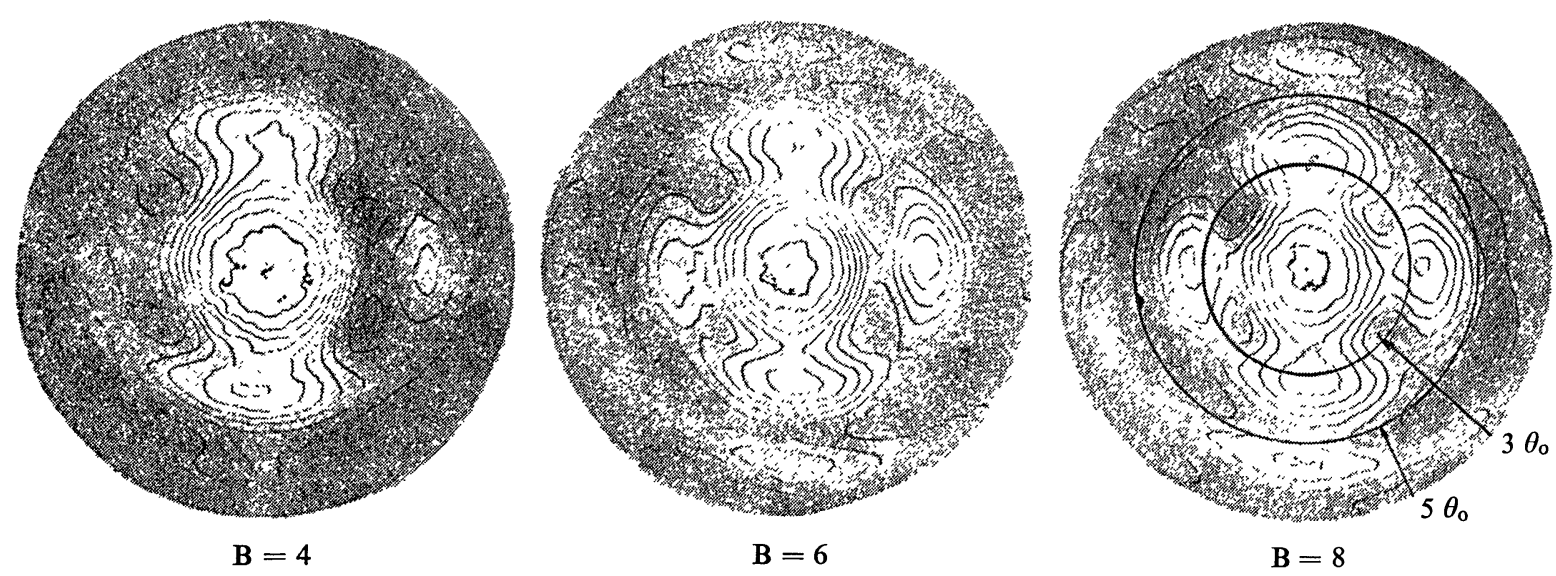

Fig. 8. - Evolution de la tache focalisée, intégrée dans le temps, en fonction de $B$.

[Variation of the time-integrated focused beam pattern vs. B.] 
Le retard de phase $\Delta \varphi$ affectant la composante moyenne $E_{0}$ du champ électrique et résultant d'une longueur de propagation $L$ dans un milieu non linéaire s'écrit :

$$
\Delta \varphi(\mathbf{r}, t)=-k \gamma F(\mathbf{r}) \cdot Q(t) \int_{0}^{L} I_{0}(z) \mathrm{d} z
$$

soit, en tenant compte de la relation (4)

$$
\Delta \varphi(\mathbf{r}, t)=-F(\mathbf{r}) \cdot Q(t) \cdot B_{0}
$$

$B_{0}$ étant la valeur de l'intégrale de rupture au centre du faisceau, à l'instant du maximum de puissance.

La composante moyenne du champ en sortie sera de la forme

$$
E=A_{0}(\mathbf{r}, L, t) \exp i\left(\phi_{0}-F(r) Q(t) B_{0}\right)
$$

et la surface d'onde sera donc variable pendant toute la durée de l'impulsion. Il en sera de même avec la répartition de la puissance dans la tache focale, résultant de la transformation de Fourier de (30). L'évolution de la densité de puissance le long d'un diamètre $x$ de la tache focale, en fonction du temps, est schématisée sur la figure 9. Si l'on suppose, qu'initialement, les distribu-

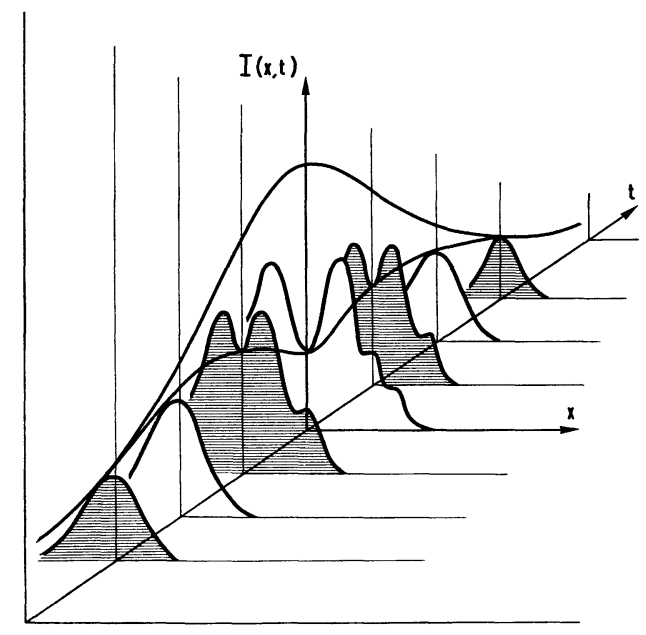

Fig. 9. - Evolution de la tache focalisée en fonction du temps. Analyse qualitative.

[Variation of the focused beam pattern vs. time. Qualitative description.]

tions spatiale et temporelle sont de forme gaussienne, la répartition de la puissance selon un diamètre $x$ de la tache focale ne restera gaussienne que pendant les premiers instants de l'impulsion durant lesquels l'intensité en champ proche est assez faible pour que $\Delta \varphi \ll 2 \pi$. Au fur et à mesure que l'intensité croît, la distorsion de la surface d'onde s'accentue et donnera une tache focale dans laquelle la puissance est rejetée du centre vers les bords, sous forme d'anneaux.

3.1 ANALYSE eXPéRIMENTALE. - L'analyse temporelle de la tache focale est effectuée au moyen d'une caméra à balayage type IMACON 600 , dont la réso- lution temporelle est d'environ 10 ps. Le dispositif expérimental est représenté sur la figure 10 : on focalise le faisceau issu d'une chaîne à barreaux, de diamètre de sortie $64 \mathrm{~mm}$, délivrant jusqu'à $250 \mathrm{GW}$ en $100 \mathrm{ps}$,

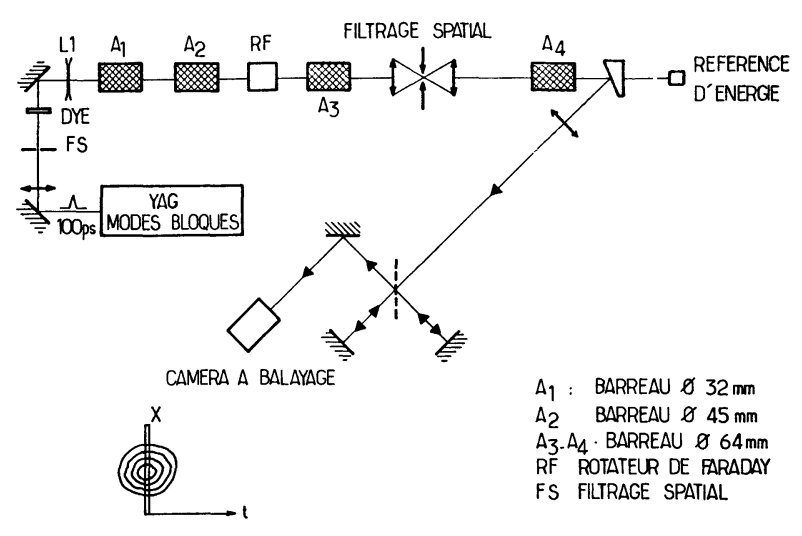

FIG. 10. - Dispositif expérimental d'analyse temporelle de la tache focalisée.

[Experimental set-up for measuring the variation of the focused energy vs. time.]

avec une valeur totale d'intégrale de rupture pouvant atteindre $B=9$. La fente d'entrée de la caméra est centrée sur la tache focale et le balayage temporel permet d'enregistrer sur film photographique l'évolution de la densité de puissance suivant un diamètre. Un exemple d'enregistrement est donné sur la figure 11 : il permet de se rendre compte qualitativement de l'évolution du phénomène.

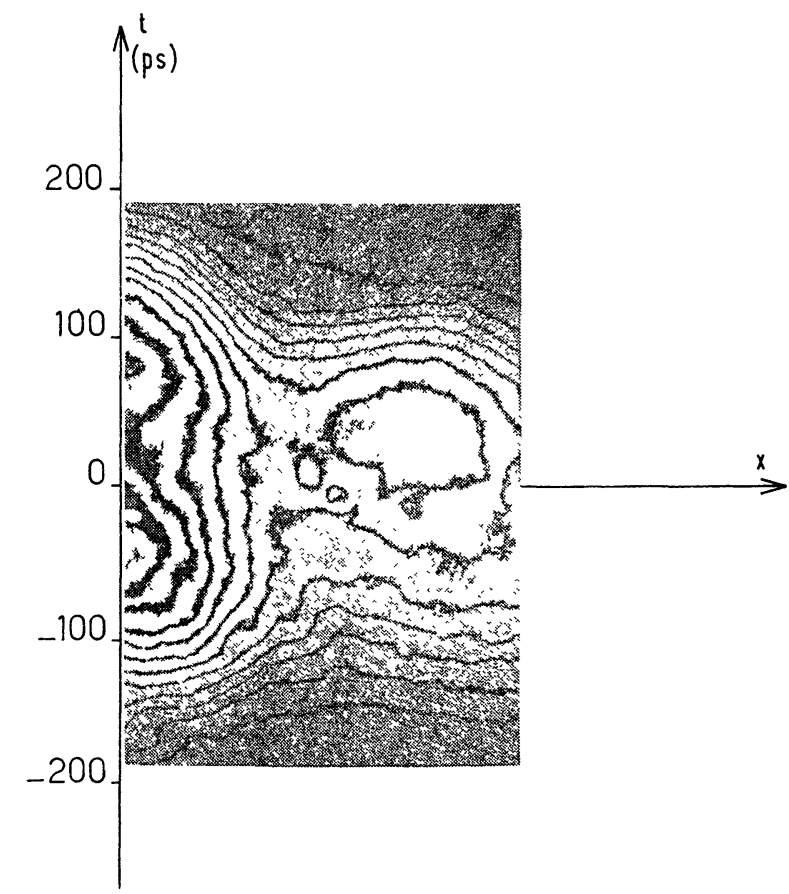

Fig. 11. - Evolution de la densité de puissance le long d'un diamètre $x$, en fonction du temps.

[Variation of the power density along a diameter $x$ vs. time.] 
L'exploitation densitométrique des enregistrements permet, après un étalonnage minutieux de la caméra et du film, de tracer l'évolution de la densité de puissance en divers points d'abscisse $x$ de la tache focale.

3.2.1 Influence de l'intégrale de rupture. - Sur la figure 12 , nous avons rassemblé les courbes d'évolution

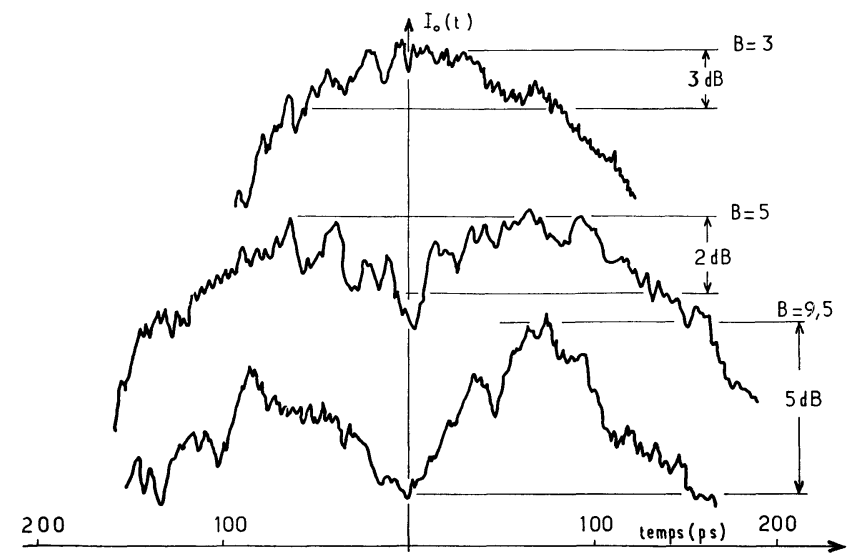

Fig. 12. - Evolution temporelle de la densité de puissance au centre de la tache focale en fonction de l'intégrale de rupture $B$ de la chaîne laser.

[Time variation of the power density at the center of the focused beam vs. the beam break-up integral $B$ of the laser chain.]

temporelle au centre de la tache focale $(x=0)$ pour 3 valeurs de $B$.

Pour $B=3$, c'est-à-dire $\Delta \varphi=0,5 \lambda$ la déformation temporelle n'est guère visible.

- Pour $B=5(\Delta \varphi=0,8 \lambda)$, la densité de puissance au centre décroît et passe par un minimum lorsque la puissance en sortie du laser est maximum.

- Pour $B=9,5(\Delta \varphi=1,5 \lambda)$, ce phénomène est encore plus renforcé.

Cette baisse de densité est le résultat d'un transfert de puissance du centre vers les bords de la tache focale et l'on peut montrer théoriquement et expérimentalement que cette puissance reste, cependant, concentrée dans un cercle de rayon $5 R_{\mathrm{d}}, R_{\mathrm{d}}$ étant le rayon minimum imposé par la diffraction. En effet, lorsqu'on mesure la puissance traversant un cercle de rayon $5 R_{\mathrm{d}}$, placé au foyer de la lentille de focalisation, on ne constate pas de distorsion temporelle (voir Fig. 13) ; la décroissance de la puissance se manifeste de façon significative seulement à l'intérieur d'une zone de rayon $3 R_{\mathrm{d}}$.

3.2.2 Influence de la répartition moyenne en champ proche. - Cette déformation temporelle est principalement le résultat de la fonction de distribution radiale $F(\mathbf{r})$ en champ proche (relation (30)). En particulier, pour une répartition moyenne uniforme $(F(\mathbf{r})=$ Cte $)$ il n'y a plus d'aberration sur la surface d'onde $(\Delta \varphi$ est

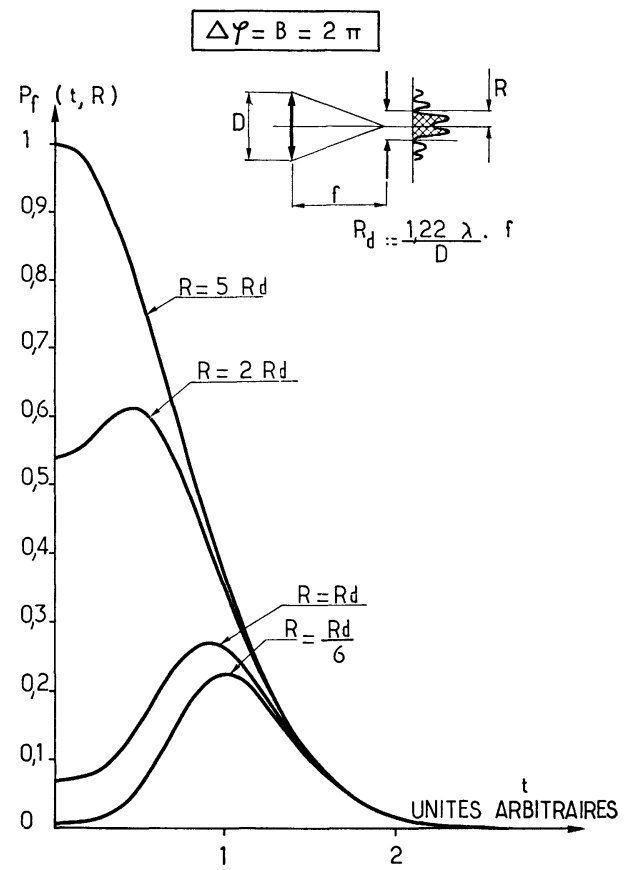

FIG. 13. - Variation de la puissance encerclée, en fonction du temps. Résultat théorique.

[Variation of power within radius $R$ vs. time. Theoretical results.]

fonction uniquement de $t$, mais non de r) et la répartition au foyer doit donc rester constante.

Expérimentalement, on peut se placer dans ces conditions en diaphragmant le faisceau en sortie du laser. La figure 14 montre les taches focales (intégrées dans le temps) obtenues avec et sans diaphragme en sortie du laser : la disparition des anneaux accompagnant la diaphragmation en champ proche démontre une amélioration de la tache focale, amélioration qui est confirmée par la variation temporelle non perturbée (Fig. 15) pour une valeur de $B=7$.

3.2.3 Influence d'un filtrage spatial. - Les résultats expérimentaux précédents ont été obtenus avec un filtrage spatial situé entre les deux étages de diamètre $64 \mathrm{~mm}$ (voir Fig. 10); le rayon du diaphragme de filtrage était supérieur à $10 R_{\mathrm{d}}$. Nous avons vu que ce diaphragme supprime efficacement les modulations à haute fréquence (Fig. 4). La puissance contenue dans ces modulations se trouve donc répartie tout autour de la tache focale principale, sur des rayons supérieurs à $10 R_{\mathrm{d}}$; elle peut atteindre $30 \%$ de la puissance totale, selon la valeur de $B$ (Fig. 7). La puissance restante est concentrée dans un cercle de rayon inférieur à $5 R_{\mathrm{d}}$ et passe donc à travers le diaphragme sans être altérée.

Le filtrage spatial réduit la puissance dispersée autour de la tache focale principale, mais il n'a pas d'action sur la répartition de la puissance focalisée. En particulier, la distorsion temporelle de la puissance au centre de la tache focale ne doit pas changer notablement lorsqu'on supprime le diaphragme du filtrage. 


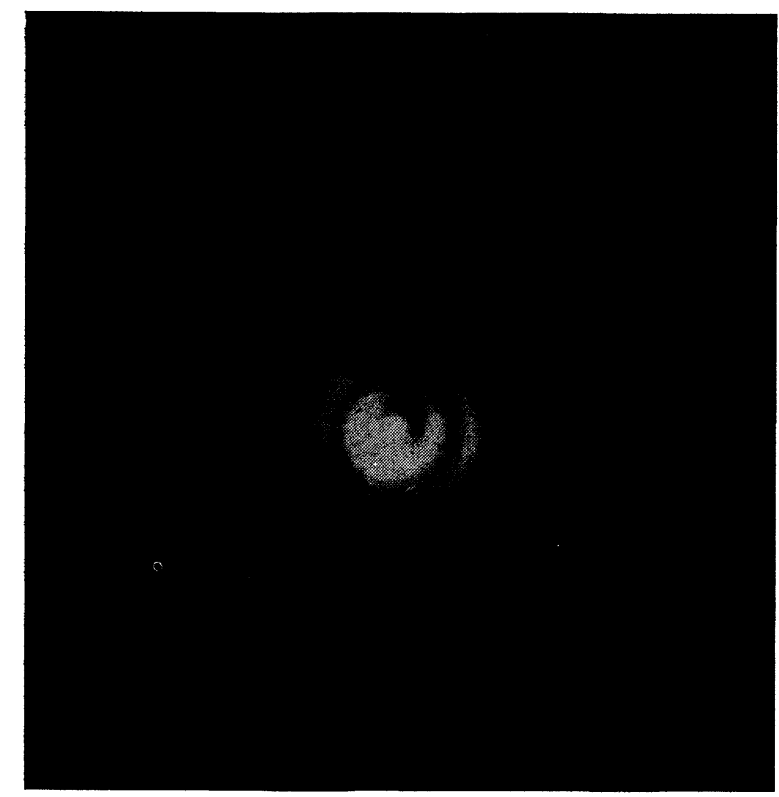

PUPILLE $\emptyset 64 \mathrm{~mm}$

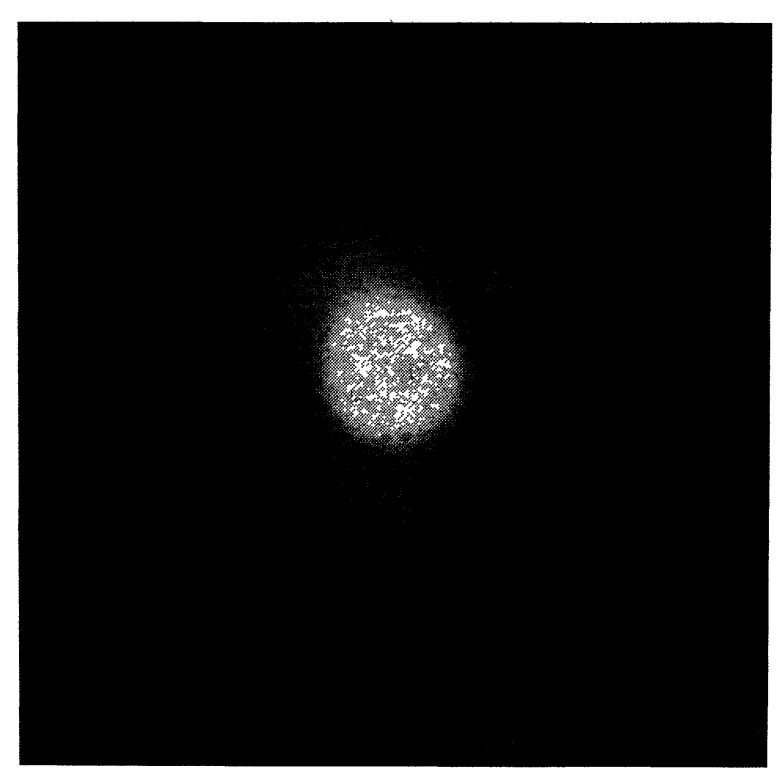

PUPILLE DIAPHRAGHE $\varnothing 20 \mathrm{~mm}$

Fig. 14. - Comparaison de la tache focale intégrée dans le temps pour une répartition moyenne uniforme en sortie du laser (faisceau diaphragmé) et pour une répartition à maximum central (faisceau non diaphragmé).

[Comparison of two time-integrated focused beam patterns corresponding to a uniform energy density profile (apertured beam) and to a bell-shaped energy profile at the laser output.]

En utilisant le même montage expérimental que celui décrit au $\S 3.2$, et en supprimant le diaphragme de filtrage, on obtient l'enregistrement de la figure 16 . Cet enregistrement est à comparer à celui de la figure 12 : pour une même valeur de $B=5$, on constate que la distorsion temporelle est très voisine dans les deux cas.

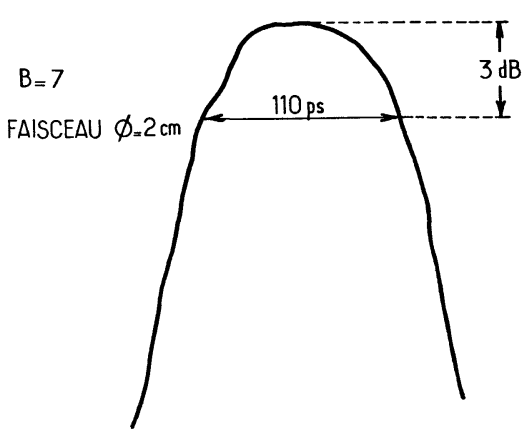

Fig. 15. - Evolution de la densité de puissance au centre de la tache focale pour une répartition moyenne uniforme en sortie du laser (faisceau diaphragmé).

[Variation of the power density at the center of the focused beam pattern for a uniform energy profile at the laser output (apertured beam).]

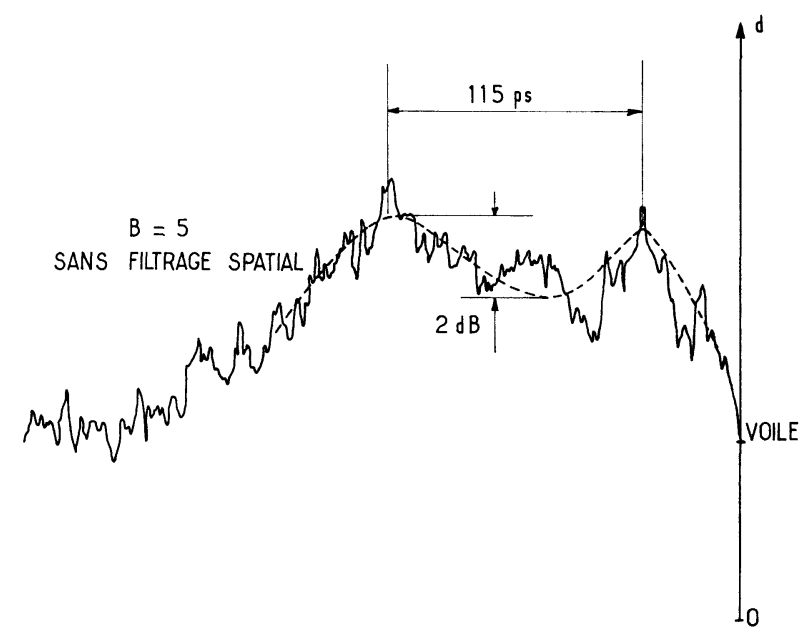

Fig. 16. - Evolution de la densité de puissance au centre de la tache focale, sans filtrage spatial dans la chaîne laser.

[Variation of the power density at the center of the focused beam pattern, without spatial filter in the laser chain.]

Par conséquent, l'effet de l'autofocalisation globale du faisceau est déterminé par la valeur totale de l'intégrale de rupture d'une chaîne et non par les valeurs de $B$ des différents tronçons situés entre filtrages.

4. Conclusions. - L'analyse des perturbations nous a montré que :

- l'amplification est impossible au-delà de $B=B_{\max }=4,5$, à moins de supprimer les perturbations par filtrage spatial ;

- la focalisation de l'onde perturbée (ou son filtrage spatial) se fait avec un rendement d'autant plus mauvais que $B$ est grand.

Pour $B=4,5$, la transmission d'un filtre spatial est de l'ordre de 0,8 ;

- l'énergie des perturbations est dispersée à l'extérieur d'un cercle de rayon $R \simeq 5 R_{\mathrm{d}}$. 
La résolution temporelle de la tache focalisée nous a montré que :

- pour des valeurs de $B>5$ la densité de puissance au centre de la tache passe par un minimum alors que la puissance est maximum en sortie du laser ;

- cette perturbation est le résultat d'un transfert de puissance du centre de la tache focale $\left(3 R_{\mathrm{d}}\right)$ vers le bord, la puissance restant néanmoins concentrée dans un cercle de rayon $5 R_{\mathrm{d}}$;

- l'importance de cette perturbation est fonction de la valeur totale de l'intégrale de rupture de la chaîne et aussi de la répartition moyenne de la puissance sur la pupille du laser ;

- dans notre configuration de chaîne, la perturbation devenait sensible pour $B=5$ (soit $\Delta \varphi=0,8 \lambda$ ) et très importante pour $B=9$ (soit $\Delta \varphi=1,5 \lambda$ ).

Ces résultats conduisent à la détermination de la structure d'une chaîne et de ses limites de puissance, compte tenu des composants utilisés :

a) Pour limiter l'effet de l'autofocalisation globale, il est nécessaire :

a) 1. soit de maintenir l'intégrale de rupture de toute la chaîne à une valeur voisine de 5 ;

a) 2. soit de disposer, dans les étages fonctionnant avec une forte valeur de $B$, d'une répartition moyenne de la puissance uniforme sur toute la section.

b) Pour limiter la modulation résultant de l'autofocalisation des perturbations, il est nécessaire de découper la chaîne en tronçons amplificateurs séparés par des filtrages spatiaux, la valeur de l'intégrale de rupture de ces tronçons étant inférieure à 4,5.

Les chaînes lasers actuelles sont construites selon ces principes. Les systèmes qui délivrent $1 \mathrm{TW}$ par faisceau, focalisable sur cible, ont des étages de sortie dont le diamètre est compris entre 150 et $200 \mathrm{~mm}$ : ces étages sont nécessairement réalisés selon la technique des disques amplificateurs qui, seule, permet de conserver, dans toute la section du matériau laser, une inversion de population convenable.

La condition imposée sur la valeur totale de B, par suite de l'autofocalisation globale, reste cependant sujette aux conditions physiques propres à la cible. En particulier, lorsque cette cible est grande par rapport à la tache limite de diffraction, on peut envisager, probablement, des conditions moins sévères sur $B$ et augmenter, par conséquent, la puissance de sortie utile du laser.

Enfin, les performances des lasers de puissance à verre au néodyme pourront évoluer notablement lorsqu'on saura réaliser des verres à faible indice non linéaire. Le verre phosphate, aujourd'hui disponible sous de grands volumes, permet d'espérer un accroissement des puissances de sortie d'un facteur proche de 2 , au moins aux impulsions très courtes, de l'ordre de 30 à 50 ps. D'autres verres sont en cours d'étude, laissant espérer ce gain de performances aux impulsions de quelques centaines de pico-secondes : l'obtention d'une puissance de $1 \mathrm{TW}$ sur un seul faisceau devient alors possible avec la technique plus classique, et moins onéreuse, des amplificateurs à barreaux.

\section{ANNEXE A}

Modèle mathématique de l'autofocalisation à petite échelle. - L'analyse des phénomènes d'autofocalisation est fondée sur l'utilisation des équations de Maxwell, dans l'approximation quasi-optique [4-12] :

$$
2 i k \frac{\partial E}{\partial z}=\nabla^{2} E+\beta|E|^{2} E .
$$

Le champ électrique total s'écrit :

$$
\begin{aligned}
& E_{\tau}=E \exp (i \omega \tau) \\
& \tau=t-z / v .
\end{aligned}
$$

Dans l'équation (A.1) $k$ est le nombre d'onde dans le vide et $\beta$ est donné par :

$$
\beta=2 k^{2} \frac{n_{2}}{n_{0}} .
$$
dire

L'opérateur $\nabla^{2}$ n'agit que dans le plan $x-y$ c'est-à-

$$
\nabla^{2}=\frac{\partial^{2}}{\partial x^{2}}+\frac{\partial^{2}}{\partial y^{2}}
$$

et la dérivée $\partial / \partial z$ est calculée pour $\tau=$ Cte.

L'enveloppe $E$ du champ électrique est de la forme

$$
E=A \exp (i \Phi)
$$

Reportant dans l'équation (A.1), on obtient deux équations :

$$
\begin{gathered}
2 k \frac{\partial A}{\partial z}=2(\nabla A) \cdot(\nabla \Phi)+A \nabla^{2} \Phi \\
-2 k A \frac{\partial \Phi}{\partial z}=\nabla^{2} A-A(\nabla \Phi)^{2}+\beta A^{3} .
\end{gathered}
$$

Ces équations ont pour solution :

$$
\begin{aligned}
& A=A_{0}=\text { Cte } \\
& \Phi=\Phi_{0}=\text { Cte }-\frac{v \beta}{2 \omega} A_{0}^{2} z
\end{aligned}
$$

c'est-à-dire l'amplitude et la phase d'une onde plane représentée par

$$
E_{0}=A_{0} \exp \left(i \Phi_{0}\right)
$$

L'onde plane subit un retard de phase proportionnel au produit intensité $x$ longueur de verre traversée.

A.I Stabilité DE L'ONDE PlAne. - On étudie l'évolution de petites variations de phase et d'amplitude de l'onde plane au moyen d'un calcul de pertur- 
bation. On recherche une solution des équations (A.7) et (A.8) qui soit de la forme :

$$
\begin{aligned}
& A=A_{0}+\bar{A} \\
& \Phi=\Phi_{0}+\bar{\Phi}
\end{aligned}
$$

où $\bar{A}$ et $\bar{\Phi}$ sont des quantités suffisamment petites pour qu'on puisse négliger leur produit ou leur carré.

Après report de (A.12) et (A.13) dans les équations (A.7) et (A.8) et linéarisation de ces équations, on obtient :

$$
\begin{array}{r}
2 k \frac{\overline{\partial A}_{1}}{\partial z}-A_{0} \nabla^{2} \bar{\Phi}_{1}=0 \\
2 k A_{0} \frac{\overline{\partial \Phi}_{1}}{\partial z}+\nabla^{2} \bar{A}_{1}+2 \beta A_{0}^{2} \bar{A}_{1}=0
\end{array}
$$

où l'écriture $\bar{A}_{1}$ et $\bar{\Phi}_{1}$ indique que l'on a seulement retenu les termes du premier ordre.

Les équations (A.14) et (A.15) ont pour solutions des expressions de la forme :

$$
\begin{aligned}
& \bar{A}_{1}=A_{0}[\delta \exp [\alpha(K) \cdot z]] H(x, y) \\
& \bar{\Phi}_{1}=-[\delta \exp [\alpha(K) \cdot z]] H(x, y)
\end{aligned}
$$

avec

$$
H(x, y)=\cos \left(K_{x} x\right) \cos \left(K_{y} y\right) .
$$

La constante $\delta$ représente la valeur initiale de l'amplitude de la perturbation

Le coefficient $\alpha(K)$ est donné par :

$$
\alpha(K)=K\left[\frac{n_{2} A_{0}^{2}}{n_{0}}-\frac{K^{2}}{4 k^{2}}\right]^{1 / 2}
$$

avec les définitions suivantes :

$K^{2}=K_{x}^{2}+K_{y}^{2}, K$ étant le nombre d'onde de la perturbation

$k=2 \pi / \lambda_{0}, k$ étant le nombre d'onde laser.
Pour des valeurs de $K$ telles que

$$
0<K^{2}<\frac{4 k^{2} n_{2} A_{0}^{2}}{n_{0}}
$$

les équations (A.16) et (A.17) indiquent que la perturbation va croître exponentiellement avec $z$. Le gain $G(K)$ est donné par :

$$
G(K)=\exp [B(K)]
$$

avec

$$
B(K)=\alpha(K) . z .
$$

On peut, en outre, dégager le point fondamental suivant : si la perturbation a une dimension privilégiée, correspondant à une fréquence spatiale critique donnée par:

$$
K_{\max }=k\left[\frac{2 n_{2} A_{0}^{2}}{n_{0}}\right]^{1 / 2}
$$

cette perturbation croîtra exponentiellement avec un coefficient de gain dont la valeur sera maximum et donné par :

$$
\alpha\left(K_{\max }\right)=\frac{\lambda_{0}}{4 n_{0} P_{1}} \cdot I_{0}=k \gamma I_{0}
$$

où $P_{1}$ est la puissance critique de Talanov, fonction de la longueur d'onde laser $\lambda_{0}$ et de l'indice non linéaire $n_{2}$ et telle que :

$$
\begin{aligned}
P_{1} & =0,950 \frac{\left[\lambda_{0}(\mu)\right]^{2}}{10^{13} n_{2}(\text { e. s. u. })} \\
\gamma & =4,19 \times 10^{-3} n_{2} / n_{0} \quad\left(n_{2} \text { en e. s. u. }\right) .
\end{aligned}
$$

A.II CAlCUl AU SECOND ORDRE. - En reportant les solutions (A.16) et (A.17) dans les équations (A.7) et (A.8), on trouve les expressions des termes du second ordre qui sont de la forme :

$$
\left.\begin{array}{l}
\bar{A}_{2}=A_{0}[\delta \exp [\alpha(K) \cdot z]]^{2} F\left[\cos \left(2 K_{x} x\right), \cos \left(2 K_{y} y\right)\right] \\
\bar{\Phi}_{2}=-[\delta \exp [\alpha(K) \cdot z]]^{2} G\left[\cos \left(2 K_{x} x\right), \cos \left(2 K_{y} y\right)\right]
\end{array}\right\} .
$$

D'après les équations (A.12) et (A.13), l'amplitude et la phase du champ électrique s'écrivent :

$$
\left.\begin{array}{l}
A=A_{0}+A_{1}+A_{2}+\cdots \\
\Phi=\Phi_{0}+\Phi_{1}+\Phi_{2}+\cdots
\end{array}\right\}
$$

On trouve, d'après les équations (A.16) et (A.17) qui prennent des valeurs maximum pour $x=y=0$, par exemple, que les équations (A.27) s'écrivent :

$\left.\begin{array}{l}A=A_{0}[1+[\delta \exp (\alpha \cdot z)]]+[\delta \exp (\alpha \cdot z)]^{2}+\cdots \\ \Phi=\Phi_{0}-[\delta \exp (\alpha \cdot z)]-\frac{1}{2}[\delta \exp (\alpha \cdot z)]^{2}+\cdots\end{array}\right\}$.

On peut donc conclure que la condition à respecter pour demeurer en deçà de l'autofocalisation, après traversée d'un milieu de longueur $L$, correspond, à la fréquence critique, à :

$$
\delta \exp \left[\alpha\left(K_{\max }\right) \cdot L\right] \leqslant 1
$$

ou

$$
\delta \exp \left[B\left(K_{\max }\right)\right] \leqslant 1
$$

\section{ANNEXE B}

Relation entre l'intégrale de rupture et les modulations d'intensité du faisceau laser. - Le champ électrique résultant du calcul de perturbation limité au premier ordre s'exprime par :

$$
E=\left(A_{0}+\bar{A}_{1}\right) \exp i\left(\Phi_{0}+\bar{\Phi}_{1}\right)
$$


où $\overline{A_{1}}$ et $\bar{\Phi}_{1}$ sont données par les équations $(\mathrm{A} .16)$ et (A. 17).

A la fréquence critique :

$$
\begin{aligned}
& \bar{A}_{1}=A_{0}\left[\delta \cdot \exp \left[B\left(K_{\max }\right)\right]\right] H(x, y) \\
& \Phi_{1}=-\left[\delta \cdot \exp \left[B\left(K_{\max }\right)\right]\right] H(x, y)
\end{aligned}
$$

avec $H(x, y)=\cos \left(K_{x} x\right) \cdot \cos \left(K_{y} y\right)$.

La densité de puissance contenue dans le faisceau laser est donnée par :

$I=E E^{*}=I_{0}\left[1+H(x, y) \cdot \delta \cdot \exp \left[B\left(K_{\max }\right)\right]\right]^{2}$

avec

$$
I_{0}=E_{0} E_{0}^{*}=A_{0}^{2} .
$$

La densité de puissance maximum (points chauds) est obtenue en des régions de l'espace telles que :

$$
K_{x} x=p \pi, \quad K_{y} y=q \pi .
$$

Elle s'écrit :

$$
I_{\max }=I_{0}\left[1+\delta \exp \left[B\left(K_{\max }\right)\right]\right]^{2} .
$$

On peut donc définir un indice de modulation $m$, tel que :

$$
m=\frac{I_{\max }}{I_{0}}=\frac{I_{0}+\Delta I}{I_{0}}=\left[1+\delta \cdot \exp \left[B\left(K_{\max }\right)\right]\right]^{2} .
$$

\section{Bibliographie}

[1] LASER Program. Annual Report. Lawrence Livermore Laboratory. UCRL 50021-74 (1974).

[2] Kelley, P. L., Phys. Rev. Lett. 15 (1965) 1005.

[3] Bespalov, V. I. and Talanov, V. I., JETP Lett. 3 (1966) 471.

[4] Suydam, B. R., Laser Induced Damage in Optical Materials (Nat. Bur. Stand. Spec. Publ. 387, Washington D. C., U. S. Government Printing Office) 1973, p. 30.

[5] Marburger, J., Jokipit, R., Glass, A. and Trendholme, J., Laser Induced Damage in Optical Materials (Nat. Bur. Stand. Spec. Publ. 387, Washington D. C., U. S. Government Printing Office) 1973, p. 49.

[6] SuydaM, B. R., IEEE J. Quantum Electron. QE II (1975) 225.

[7] Buiss, E. S., Sommargren, G. E. and Weawer, H., Digest of technical Papers (IEEE/OSA Conference on Laser
Engineering and Applications, Washington D. C.) 1975 , p. 29.

[8] Bliss, E. S., Speck, D. R., Holzrichter, J. F., ErkKIla, J. H. and Glass, A. J., Appl. Phys. Lett. 25 (1974) 448.

[9] Robieux, J., Guyot, J., Bettinger, A., Auric, D., 3e Rapport Quadrimestriel (Contrat CEA/DAM 2999/E, Laboratoires de Marcoussis) 1974.

[10] Robieux, J., Guyot, J., Bettinger, A., Auric, D., 2e Rapport Quadrimestriel (Contrat CEA/DAM 3035/E, Laboratoires de Marcoussis) 1975.

[11] Auric, D., Labadens, A. and Guyot, J., Conference Digest (9th International Conference on Quantum Electronics, Amsterdam) 1976, p. 175.

[12] Akhmanov, S. A., Laser Handbook. Edité par F. T. Arecchi et E. O. Shulz-Dubois (North-Holland Publ. Co) 1972 , p. 1152. 